\title{
Family caregiving for persons with heart failure at the intersection of heart failure and palliative care: a state-of-the-science review
}

\author{
J. Nicholas Dionne-Odom ${ }^{1} \cdot$ Stephanie A. Hooker $^{2}$ - David Bekelman ${ }^{3}$ • Deborah Ejem ${ }^{1}$. \\ Gwen McGhan $^{1} \cdot$ Lisa Kitko $^{4}$ - Anna Strömberg ${ }^{5} \cdot$ Rachel Wells $^{1}$ • Meka Astin ${ }^{6}$. \\ Zehra Gok Metin $^{7}$ - Gisella Mancarella ${ }^{1}$ - Salpy V. Pamboukian ${ }^{8}$. \\ Lorraine Evangelista ${ }^{9} \cdot$ Harleah G. Buck ${ }^{10}$ - Marie A. Bakitas ${ }^{1} \cdot$ On behalf of the \\ IMPACT-HF National Workgroup
}

Published online: 4 February 2017

(C) Springer Science+Business Media New York 2017

\begin{abstract}
Many of the 23 million individuals with heart failure (HF) worldwide receive daily, unpaid support from a family member or friend. Although $\mathrm{HF}$ and palliative care practice guidelines stipulate that support be provided to family caregivers, the evidence base to guide care for this population has not been comprehensively assessed. In order to appraise the state-of-the-science of HF family caregiving and recommend areas for future research, the aims of this review were to summarize (1) how caregivers influence patients, (2) the consequences of HF for caregivers, and (3) interventions directed at HF caregivers. We reviewed all literature to December 2015 in PubMed and CINAHL using the search terms "heart failure" AND "caregiver." Inclusion criteria dictated that studies re-
\end{abstract}

Electronic supplementary material The online version of this article (doi:10.1007/s10741-017-9597-4) contains supplementary material, which is available to authorized users.

J. Nicholas Dionne-Odom

dionneod@uab.edu

On behalf of the IMPACT-HF National Workgroup

1 School of Nursing, University of Alabama at Birmingham, 1720 2nd Avenue South, 2M019H, Birmingham, AL 35294-1210, USA

2 Department of Psychology, University of Colorado, Denver, Campus Box 173, PO Box 173364, Denver, CO 80217, USA

3 Veterans Affairs Eastern Colorado Health Care System, Department of Medicine, Anschutz Medical Campus, University of Colorado School of Medicine, 1055 Clermont Street, Research 151, Denver, CO 80220, USA

4 College of Nursing, Pennsylvania State University, 201 Nursing Sciences Building, University Park, PA 16802, USA port original research of HF family caregiving. Articles focused on children or instrument development or aggregated HF with other illnesses were excluded. We identified 120 studies, representing 5700 caregivers. Research on this population indicates that (1) caregiving situations vary widely with equally wide-ranging tasks for patients to help facilitate their health behaviors, psychological health and relationships, and quality of life (QoL); (2) caregivers have numerous unmet needs that fluctuate with patients' unpredictable medical status, are felt to be ignored by the formal healthcare system, and can lead to distress, burden, and reduced QoL; and (3) relatively few interventions have been developed and tested that effectively support HF family caregivers. We provide recom-

5 Department of Medical and Health Sciences, Division of Nursing, Campus University Hospital, Linköping University, Building 511-001, 58185 Linköping, Sweden

6 School of Public Health, University of Alabama at Birmingham, 1720 2nd Avenue South, Birmingham, AL 35294, USA

7 Department of Internal Medicine Nursing, Hacettepe University, 06100 Ankara, Turkey

8 Division of Cardiovascular Diseases, Department of Medicine, University of Alabama at Birmingham, 1900 University Blvd, THT 321, Birmingham, AL 35294, USA

9 Program in Nursing Science, University of California, Irvine, 299E Berk Hall, Irvine, CA 92697-3959, USA

10 College of Nursing, University of South Florida, 12901 Bruce B. Downs Blvd. MDC22, Tampa, FL 33612-4766, USA 
mendations to progress the science forward in each of these areas that moves beyond descriptive work to intervention development and clinical trials testing.

Keywords Heart failure $\cdot$ Family caregiving $\cdot$ Palliative care

\section{Introduction}

Support provided by family caregivers is critical to maintaining the health and quality of life $(\mathrm{QoL})$ of the over 23 million individuals worldwide affected by heart failure (HF) [1]. Having HF is associated with functional impairment [2]; debilitating symptoms [3]; and complex medication, device, and self-care regimens $[4,5]$ that often require the regular assistance of unpaid family caregivers. Several Institute of Medicine (IOM) reports have described the toll that performing tasks for persons with serious illnesses can have on family caregivers' health and well-being, which may consequently hinder their ability to provide high quality care to patients [6-9]. These IOM reports stress the imperative of ensuring that family caregivers are kept healthy and functioning, particularly in the advanced stages of illness when both patient care demands and the risk of caregiver burden increase. Among patients with advanced HF, specialist palliative care clinicians play an important role for HF caregivers because a core component of palliative care practice is the assessment of family caregivers and the development of care plans that specifically address caregivers' unique needs [10]. Moreover, a recent American Heart Association and American Stroke Association policy statement [11] stress the importance of including caregivers in palliative HF treatment to assist with managing patients' complex needs and thereby reduce caregiver burden.

Despite an increased general awareness of the need to support family caregivers, the extent and quality of research and the evidence base that it has produced to guide clinical care of this population has yet to be comprehensively assessed. Several reviews have evaluated particular aspects of HF family caregiving, including general experiences [12], relationship quality [13], needs [14], burden [15, 16], roles in patient self-care [17-19], and left ventricular assist device management [20]. However, to our knowledge, the evidence base of original research focused on HF family caregiving has yet to be assessed in its entirety in order to inform future research directions and priorities in HF palliative care. Accordingly, the aims of this state-of-the-science review were to (1) summarize caregivers' impact on patient outcomes, HF's impact on caregiver outcomes, and HF interventions involving caregivers; and (2) provide a summary of future research priorities in palliative care for HF family caregivers.

\section{Methods}

Data sources, search strategies, and study selection

We conducted an integrative narrative review [21] of original research focused on family caregivers of adults with $\mathrm{HF}$ for all months and years up to December 2015. We searched PubMed and CINAHL using the search string: "caregiver" AND "heart failure" AND hasanabstract[text] AND English[lang]. Eligibility criteria to screen abstracts included the following: original research of qualitative, quantitative, mixed method, or intervention research; published in English; and reported data collected from family caregivers. Articles excluded were as follows: focused on children and the pediatric context, included HF caregivers in aggregate with other serious or chronic illnesses (e.g., cancer, dementia), or focused on instrument development. Conference abstracts, systematic reviews, letters to the editor, commentaries, and research that included paid or professional caregivers were also excluded.

The initial search identified 768 articles (Fig. 1). After removing duplicates $(n=114)$, two authors (JND-O, ZGM) screened the titles and abstracts of 654 articles. Articles were retained if they met the eligibility criteria. This resulted in 174 articles that were assessed for eligibility based on full-text review. After discussion to resolve disagreements, 120 articles were included for analysis.

\section{Data extraction, synthesis, and analysis}

Four co-authors (SH, DE, MA, RW) and three undergraduate research assistants independently read eligible articles and abstracted data on the following: study design; objective/aims; hypothesis (if applicable); theory or conceptual framework; caregiver and patient sample size and eligibility criteria; variables, instruments, and measurement frequency (quantitative only); qualitative methodology and analysis approach; main findings; clinical implications; and study limitations. The first author (JND-O) reviewed each article abstraction for accuracy and completeness. Disagreements were discussed until consensus was reached. Articles were then categorized as addressing one or more of the following topical areas based on studies' research objectives and objectives/ aims: (1) caregiver impact on patients, (2) HF's impact on caregivers, and (3) caregiver interventions. Following the approach of an integrative narrative review, data were then composed into narrative summaries that summarized topics, methods, and general patterns of results [21]. For a brief description of each article, see the online Supplemental Table. 


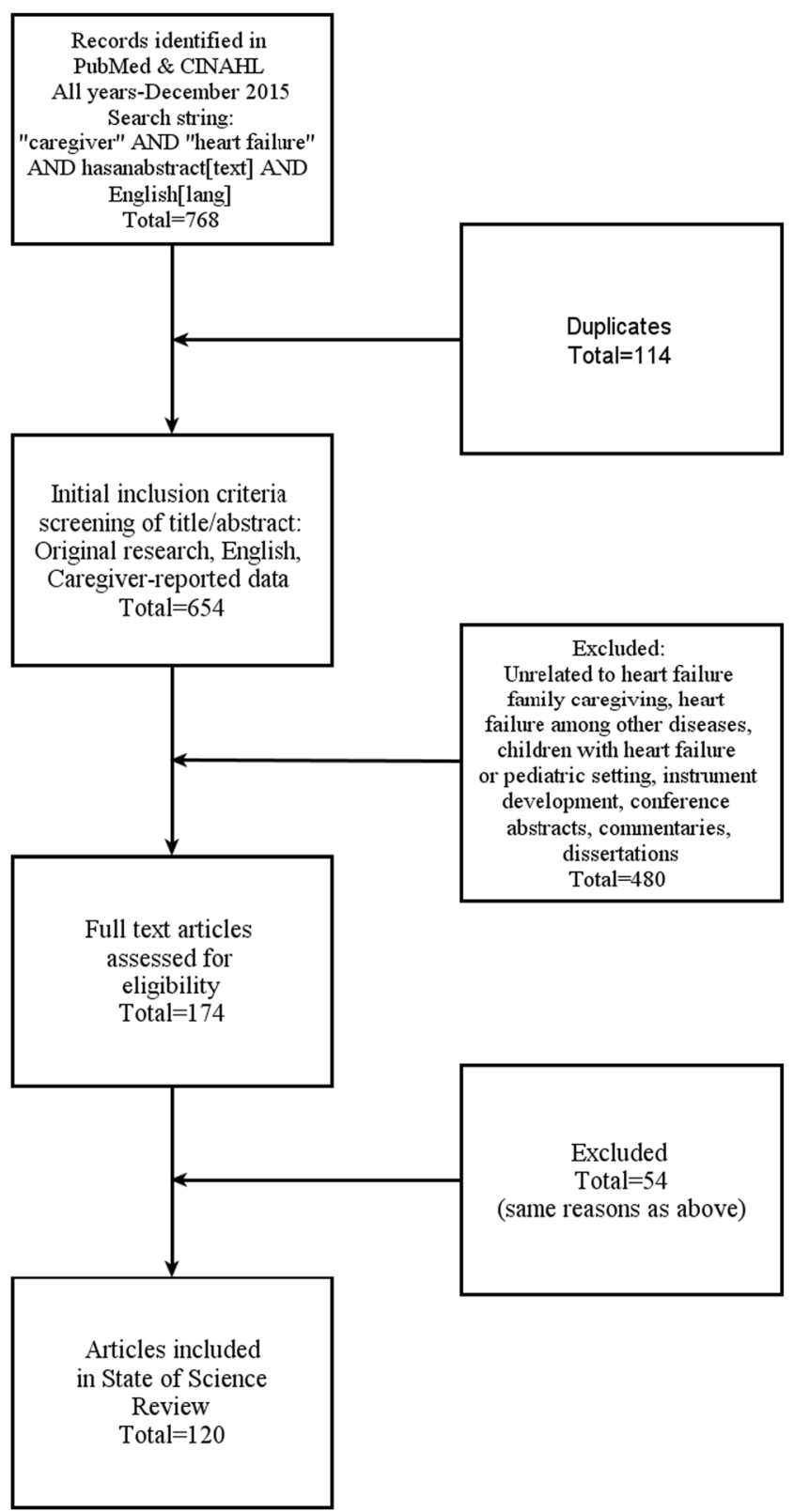

Fig. 1 Summary of literature search and selection

\section{Role of funding sources}

This review did not receive any direct funding support and was conducted on behalf of a national working group, Improving Palliative Care Therapies for Heart Failure Patients and Families (IMPACT-HF ${ }^{2}$ ). The mission of IMPACT-HF ${ }^{2}$ is to gather leading palliative care and HF experts to set priorities for future research, policy, and practice. IMPACT-HF ${ }^{2}$ has received funding support from John A. Hartford Foundation Centers of Excellence in Geriatric Medicine and Geriatric Psychiatry Collaborative Pilot Project, the University of Alabama at Birmingham, the Icahn School of Medicine at Mount Sinai, and the National Palliative Care Research Center; however, none of these entities had a role in the design or conduct of this study or in the decision to submit the manuscript for publication.

\section{Results \\ Overview}

Our literature search yielded 120 articles published in English before 31 December 2015 that met the eligibility criteria [22-141]. These articles reported on data from 97 unique datasets. As shown in Fig. 2, the first study was published in 1994 and since that time, an increasing number of studies have been published. Publication and study characteristics are listed in Table 1. The number of HF family caregivers in study sample sizes ranged from 4 to 415 , for a total of 5754 caregivers. Of studies reporting the relevant demographic characteristics $(\mathrm{k})$, the mean age of caregivers was $55.9(\mathrm{k}=76)$ and the majority were female $(\mathrm{k}=70.2 \%, \mathrm{k}=83)$, white $(71.3 \%$, $\mathrm{k}=43$ ), with some college or higher educational level $(41.5 \%$, $\mathrm{k}=37)$, and spouses $(65.2 \%, \mathrm{k}=77)$ of the individual with HF. Thirty-seven studies $(30.1 \%)$ did not report any caregiver demographic characteristics, and 25 did not state any eligibility criteria for caregivers. The majority of studies reported qualitative data $(\mathrm{k}=45)$. For nonqualitative studies, the most commonly measured caregiver outcomes were QoL, general health, burden, and depressive symptoms (Table 2). A single point of data collection occurred in 51 studies, and 26 had follow-up measures ranging from 4 weeks to 24 months after the first measurement.

\section{How caregivers influence patients}

Almost one third of the articles $(\mathrm{k}=33)$ reported results on how family caregivers impact patients. Major areas of focus included patient health behaviors, psychological health and relationships, QoL, and healthcare utilization.

\section{Caregivers' influence on patients' health behaviors}

Twenty-two studies focused on family caregivers' roles and impact on supporting care recipient practices to improve and maintain healthy behaviors. Qualitative $[39,53,54,65,85$, $88,109,116]$ and mixed method $[22,68]$ studies reported that family caregivers assisted individuals with numerous health behaviors, including managing and administering medications; assisting with healthcare communication and coordination; staying physically active; eating healthy and cooking; expressing and managing emotions; getting adequate rest; offering spiritual support; helping with personal hygiene, such as bathing and getting dressed; managing the home; and monitoring, treating, and coping with symptoms. 
Fig. 2 Number of articles over time by research design

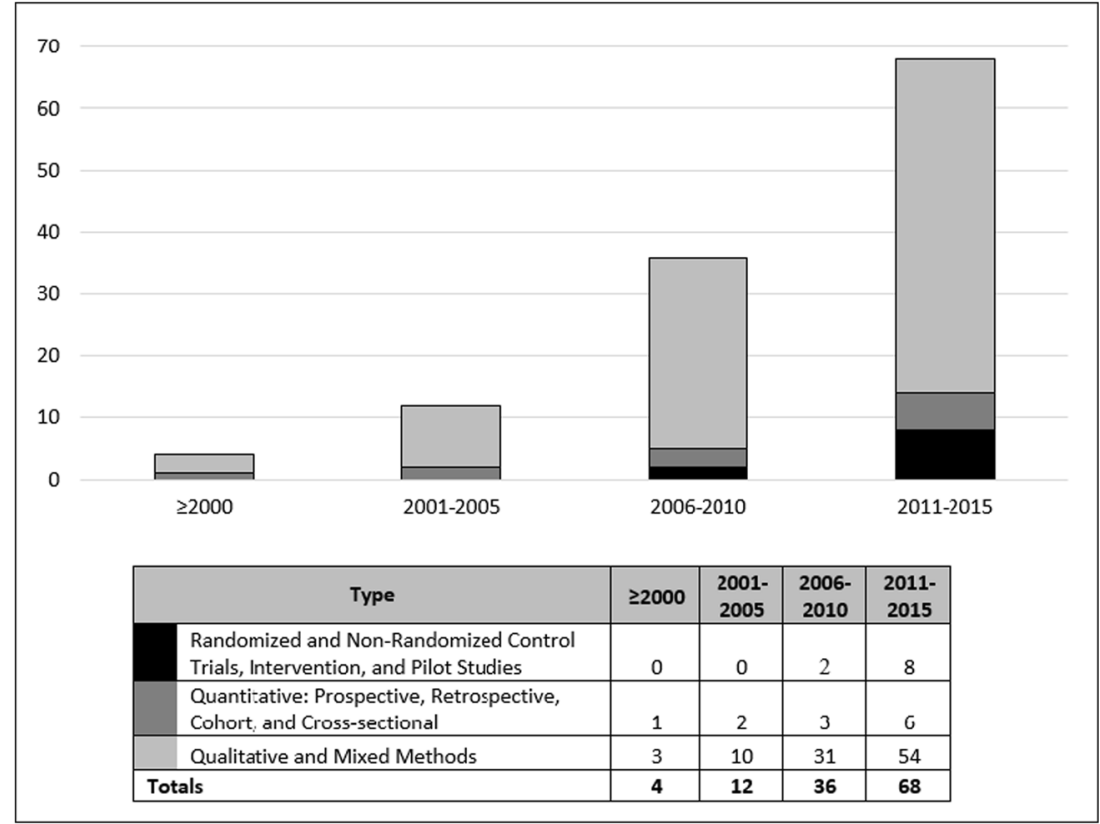

These qualitative and mixed method studies reported that most patients found the caregivers' support of their health

Table 1 Publication and study characteristics

\begin{tabular}{ll}
\hline Characteristic & No. $(\%)$ \\
\hline Total no. of articles & 120 \\
Unique datasets & 97 \\
Study setting by country & \\
$\quad$ USA & $67(55.8 \%)$ \\
Europe (excluding the UK) & $21(17.5 \%)$ \\
UK & $16(13.3 \%)$ \\
Iran & $6(5.0 \%)$ \\
Canada & $6(5.0 \%)$ \\
Australia & $2(1.7 \%)$ \\
Turkey & $1(1.0 \%)$ \\
Taiwan & $1(1.0 \%)$ \\
Study design type & \\
Qualitative & $45(37.5 \%)$ \\
Quantitative & $44(36.7 \%)$ \\
RCT & $10(8.3 \%)$ \\
Mixed methods & $8(6.7 \%)$ \\
Cohort & $7(5.8 \%)$ \\
Nonrandomized & $6(5.0 \%)$ \\
Sample size, total all studies & 5754 \\
Mean age, total all studies & 55.9 \\
Gender, female, total all studies & $3838(70.2 \%)$ \\
Race, white, total all studies & $1640(71.3 \%)$ \\
Studies reporting no demographic data & $37(30.8 \%)$ \\
\hline & $25(20.8 \%)$ \\
\hline Studies reporting no eligibility criteria & \\
&
\end{tabular}

behaviors to be helpful; however, some articles described conflicting views about the help that family members provided. For example, when 100 patient and caregiver dyads were interviewed, almost half were incongruent on their perspectives towards illness management, healthcare issues, and endof-life decisions [85]. In addition, a study of 30 family caregivers reported that many caregivers lacked clinical knowledge and understanding about HF and health behavior management, particularly around symptom exacerbation management and diet [54]. In a study of congruence between 70 patients and their caregivers on symptom and self-care assessment, caregivers and patients generally agreed on self-care management; however, caregivers were relatively poor at judging patients' feelings of depression [115]. Poor caregiver self-care "modeling behaviors" have also been identified as a barrier to patient health behaviors; for example, one study quoted a patient mentioning that her "husband likes fried chicken... [which] throws us off" because when he brings unhealthy food home, she eats it with him [68].

Most quantitative studies identified family caregiver characteristics, such as caregiver physical health, strain, mental health, ability to partner in decisions, and health literacy, that were associated with patient health behaviors. One study identified poor caregiver health and higher strain as associated with patients having higher confidence in providing themselves self-care [86]. Similarly though in the opposite direction, in an Italian sample of 364 dyads, increased caregiver physical QoL was associated with lower levels of patient selfcare management for male patients [37]; male patients who had healthier caregivers were less likely to personally attend to their self-care needs. The authors postulated that poorer selfcare behaviors among male patients may be a function of poor 
Table 2 Most common study outcome constructs and measures

\begin{tabular}{lll}
\hline $\begin{array}{l}\text { Outcome } \\
\text { construct }\end{array}$ & Measure & $\begin{array}{l}\text { No. of studies employing } \\
\text { measure }\end{array}$ \\
\hline Quality of life & Quality of Life Index & 3 \\
& Bakas Caregiving Outcomes Scale & 3 \\
General Health & SF-36 & 8 \\
& SF-12 & 8 \\
& Rand 35 Physical Health & 4 \\
Burden & Zarit Caregiver Burden Interview & 8 \\
& Caregiver Reaction Assessment & 7 \\
& Objective Burden Inventory & 4 \\
& Oberst Caregiving Burden Scale & 4 \\
Depression & Center for Epidemiological Studies Depression & 8 \\
& Scale & 5 \\
& Patient Health Questionnaire-9 & 3 \\
\hline
\end{tabular}

symptom recognition. In contrast to caregiver physical health and strain, multiple studies showed that better caregiver mental health [44, 95, 133-135] and their ability to partner in decisions [128] was associated with increased patient confidence and maintenance of their self-care practices. Caregiver health literacy has also been associated with patient self-care behaviors, albeit only one study was found addressing this topic; in that study, caregivers who had higher label-reading skills were more likely to have patients who were better able to perform HF self-care behaviors [87].

\section{Caregivers' influence on patients' psychological well-being}

Eight studies addressed the potential impact that family caregivers have on patients' psychological health and relationships [43, 46, 63, 85, 98, 115, 116, 133]. Qualitative studies focused on the relational dynamics between patients and their caregivers $[43,85,116]$. Some studies examined congruence between patients and caregivers about how to manage and cope with HF $[83,116]$. These studies noted that incongruent dyads experienced marked relational tension and psychological distress.

Three quantitative studies reported mixed findings between psychological and social health outcomes (e.g., depression and perceived social support) of patients and family caregivers $[63,98,133]$. One study found that caregivers' emotional well-being accounted for $54 \%$ of the variance in their care recipients' emotional well-being [63]. Similarly, in a sample of 23 spousal dyads, increased levels of caregiver depressive symptoms and burden were associated with worse patient depressive symptoms and decreased patient-perceived social support and relationship satisfaction [133]. In contrast, in a sample of 48 dyads where the patients were all male, spousal health-related QoL and depression did not influence these male patients' depressive symptoms [98].

\section{Caregivers' influence on patients' quality of life}

Five quantitative studies specifically focused on the influence that family caregivers have on patients' QoL and physical health. In two studies, increased family caregiver confidence in providing self-care was associated with worse patient physical QoL [134, 135]. Caregiver confidence was demonstrated to moderate the relationship between a patient's self-care and their physical QoL. The authors discussed that as the physical health of the patient declines, the caregiver must assume more of the patients' self-care responsibilities; hence, as a patient's QoL becomes poorer, a caregiver's confidence might rise as they assume more responsibility, which was validated by another study evaluating this question [127]. They reported that caregivers who reported higher esteem were more likely to care for patients who reported lower health-related QoL. Two studies also showed that higher caregiver burden and depression were associated with poorer patient physical health and QoL [51, 69].

\section{Caregivers' influence on patients' healthcare utilization}

Four studies reported associations between family caregiving and patients' healthcare utilization [29, 70, 122, 125]. A qualitative study of ten African-American patient-caregiver dyads explored caregiver decision-making around a hypothetical scenario of going to hospital for worsening heart failure symptoms and found that caregivers often perceived themselves to have a critical role in those decisions [70]. Two quantitative studies reported caregiver perceptions of low social support 
[122], higher care hours [122], and worse depression and stress [125] predicted more frequent patient hospitalizations.

\section{The consequences of heart failure for caregivers}

Over two thirds $(\mathrm{k}=81)$ of the 120 articles reported on how HF impacts family caregivers. Major topics included the following: the HF illness trajectory for caregivers; caregivers' unmet needs; left ventricular assist devices (LVADs); and caregiver psychological well-being, burden, and QoL.

\section{Heart failures' effects on caregiver experiences over time}

Thirteen studies addressed how fluctuations in the HF illness trajectory impact caregiving experiences over time $[39,57$, $72,73,77,78,84,88,97,113,118,127,140]$. Qualitative studies $[39,72,73,77,78,84,88,97,118,140]$ described a number of similar themes including the following: initial doubts and anxiety about performing in the caregiving role that improved over time; frenzied periods of medical instability (e.g., unanticipated emergency visits to hospital, device placement, heart transplant) followed by medical stability and concerted efforts to stabilize to a normal lifestyle; need for constant guidance and social support; persistent worry and stress over uncertainty about the future; constant "juggling" of work, home, and caregiving tasks; increased sense of responsibility and role in decision-making as care recipients health and functional ability worsened over time; constant adoption of new strategies and adaptations to normalize life as much as possible; feeling more socially isolated over time as care tasks increased; and a mixture of acceptance, relief, and sadness as end of life neared.

\section{Resultant unmet needs of heart failure family caregivers}

Twenty-nine studies sought to characterize what caregivers perceived as their key needs when supporting someone with HF. Qualitative studies [30, 36, 40, 42, 45, 48, 54, 61, 71, 72, $74,77,80,83,88,104,110,117,119,131,137,138,142]$ described a wide range of needs that caregivers felt underprepared for or in need of assistance with, including coping with and managing symptoms; understanding HF and prognosis; handling unplanned hospitalizations and other emergencies; managing medications and devices; providing and receiving emotional and spiritual support; partnering in patients' selfcare; balancing home and work; engaging in their own selfcare; having difficult conversations; handing uncertainty about the future and the unpredictably of the disease; communication and care coordination; understanding palliative care services; managing the home; dealing with financial issues; knowing what to expect and how to care for patients at end of life; and leveraging and timely access to formal and informal social support and services. Two quantitative studies both identified handling emergencies as a situation that caregivers' felt most unprepared for [127, 136].

Several qualitative studies noted that caregivers stated feeling that these unmet needs were ignored by the formal healthcare system, which further intensified the strain and burden of the role and engendered distrust of the healthcare system. In one of these studies [48], caregivers expressed this type of distrust in the healthcare system when they felt the role they were expected to fulfill exceeded or was incompatible with their ability to perform that role. Frustration with healthcare providers providing a lack of information was another theme identified in another study in which "providers conveyed a sense that they were busy, and partners [caregivers] said they had few opportunities to ask questions and to get them answered in an understandable manner" [77].

\section{Unique experiences of caregivers of patients with left ventricular assist devices}

Six qualitative $[27,33,60,84,97,99]$ and three mixedmethod studies $[34,38,82]$ examined the roles and experiences of family caregivers in the context of their care recipients having a left ventricular assist device (LVAD). Several studies focused on the caregiver's role in decision-making before LVAD implantation. LVAD candidates stressed the importance of an informed caregiver to help synthesize information received during the decision-making process [38]. Caregivers reported the decision-making process as stressful [82] with "tensions" during the process between wanting their loved one to live but yet anxiously anticipating the host of challenges that will accompany the decision to receive an LVAD [99].

Other studies focused on the caregiver's experience after implantation [27, 33, 82, 84, 97]. Caring for a patient with an LVAD included simple tasks such as monitoring vital signs, daily weights, and sterile dressing changes and also more complex procedures, such as monitoring the LVAD driveline, troubleshooting alarms, and responding to emergency situations. Performing these tasks was stressful for caregivers and many reported not being psychologically prepared for their new role as they described the post-implantation period being filled with worry and stress. Despite initial anxieties, a common theme reported by caregivers in these studies was successful adaptation and acceptance of their new role over time.

\section{Heart failure's influence on caregivers' psychological well-being, burden, and quality of life}

Forty-six studies examined the impact of HF on family caregivers' psychological well-being, burden, and QoL. Quantitative studies identified a variety of factors associated with worse caregiver psychological well-being (e.g., depression, anxiety, stress). Traits of caregivers themselves included: 
younger age, being female, being unmarried, being a child of the patient, having an anxious preoccupation coping style, and having low health-related QoL, high burden, poor functional status; and low self-care confidence [52, 59, 92, 105, 121, 134, 141, 143, 144]. Characteristics of the caregiving context and the patient included higher number of caregiving hours and tasks, low sense of perceived control, poor relationship quality with patients, low familial social support, poor family functioning, and caring for patients who are younger, lack employment, depressed, have a high number of symptoms, and exhibit poor self-care maintenance behaviors [49, 52, 59, 81, 90, 98, 103, 105, 113, 124, 133, 134, 143].

Quantitative studies also identified factors associated with caregiver burden. Traits of caregivers themselves included older age, being female, being white, and having lower education and experiencing depressive symptoms, poor physical health, poor mental health, and low spiritual well-being [24, $32,93,96,123,124,139,141]$. Characteristics of the caregiving context and the patient included higher hours of caregiving, caring for multiple persons, poor care continuity, poor relationship quality with the patient, low perceived social support, financial strain, poor patient health; lower sense of control, dealing with patient behaviors, patient depressive symptoms, patients with poor functional status and/or high activities of daily living dependence $[32,57,69,75,90,93,102$, $123,133,139]$. Joo and colleagues [79] assessed the economic burden of HF family caregiving and found that caregivers provided an average of $32.1 \mathrm{~h} /$ week of support and a cost of $\$ 3$ billion in 2010; however, others have reported both higher [113] and lower [66] estimates of caregiving hours/week. A study by Davidson and colleagues [56] found that HF family caregivers provided care for an average of 48.9 months.

Finally, quantitative studies examined variables associated with worse caregiver QoL and physical health, including being female; having low perceived control, social support, and self-esteem; having symptoms of depression and/or anxiety, poor medical health including mental health, perceived difficulty in caregiving tasks; and caring for patients with a high number of symptoms and poor prognosis $[23,51,75,92,94$, $113,121]$.

Qualitative [28, 30, 41, 61, 77, 80, 85, 91, 106, 116, 117] and mixed-method $[35,82]$ studies explored a number of possible caregiver-identified causes responsible for their distress and burden. These included lack of care-related knowledge, physical exhaustion from performing caregiving tasks, exhaustion from communication and providing psychosocial support, dealing with care recipients who were nonadherent to healthy self-care practices, always having to be ready to step-in, struggling to maintain a "normal" daily life and work responsibilities, worrying about the future, not being able to do what they used to, less sexual intimacy, social isolation, poor social support, and poor health professional support.

\section{Interventions directed at heart failure family caregivers}

Sixteen studies evaluated interventions targeted at HF family caregivers. Ten studies had a randomized controlled trial design $[25,26,62,89,100,101,110,112,126,130]$ and six were quasi-experimental, nonrandomized designs $[50,55,58$, $107,111,129]$. Sample sizes ranged from 11 to 369 caregivers, though the majority $(n=11)$ had less than 70 caregiver participants, including 5 of the 10 randomized trials. Most caregivers in these trials $(n=1061)$ were female $(78.0 \%)$ with a mean age of 54.9 and were the patient's spouse or partner $(65.0 \%)$ or their children $(29.8 \%)$.

There were a wide range of caregiver intervention durations, designs, and delivery methods. Intervention duration ranged from 1 to 12 months. Outcomes were followed up to 24 months after baseline. Most studies applied a dyadic approach involving both the patient and caregiver in the intervention $[25,26,55,58,101,110,111,126,129,130]$, while others focused solely on the caregiver $[50,62,89,100,110]$. Five interventions $[25,26,50,100,130]$ involved individual nurse-led face-to-face psychoeducational sessions sometimes in combination with other approaches to follow-up such as home visits [25, 100], telemonitoring [50], and telephone calls $[100,130]$. Notably, none of the 16 intervention studies involved specialist palliative care clinicians or addressed advance care planning. Four studies provided a tele- or mhealth interventions alone: two using telephone coaching [58, 110], one using telemonitoring [126], and one using weekly automated self-care support calls and notifications about problems sent to clinicians alone or in combination with automatically generated emails sent to the caregivers with suggestions for self-care assistance [111, 112]. Two studies employed a support group format; one led by a nurse (4 sessions) [62] and the other by a member of a multidisciplinary team (6 sessions) [89]. Two other studies [55, 129] tested interventions consisting of in-person psycho-educational and skill-building small group sessions involving caregivers and patients, both together and separately in different sessions. One study tested whether an exercise intervention for patients impacted caregiver outcomes without there being a specific intervention component for the caregiver [101].

Primary caregiver-reported outcomes assessed and instrumentation varied widely among trials. Outcomes included caregiver burden $(n=10)$, depressive symptoms $(n=7)$, QoL $(n=5)$, anxiety symptoms $(n=4)$, caregiving preparedness $(n=3)$, caregiving confidence $(n=2)$, relational quality with patient $(n=2)$, self-care $(n=2)$, general health $(n=1)$, perceived control $(n=1)$, mastery of stress $(n=1)$, satisfaction ( $n=1)$, caregiver knowledge $(n=1)$, family functioning $(n=1)$, and assistance with patient self-care $(n=1)$. Four trials evaluated the interventions' impact primarily on the patient outcomes [100, 110, 126, 130]. 
Of the eight randomized trials that included primary caregiver outcomes, only three studies reported a statistically significant positive effect from their intervention on the primary caregiver outcomes $[62,110,112]$. All three showed a statistically significant reduction in caregiver burden. Piette and colleagues' [112] mobile health support intervention to help caregivers promote self-management showed a reduction of depressive symptoms in caregivers at 6 and 12 months. Nurseadministered coaching sessions by telephone significantly improved caregiver confidence and preparedness [110]. Of the two randomized trials only having patient outcomes [126, 130], one had positive outcomes: Shahriari and colleagues' [130] three-session group educational intervention demonstrated an increased number of patient self-care behaviors at 1 month.

\section{Discussion}

We conducted the first comprehensive assessment of the research evidence base focused on family caregiving for individuals with HF. Our effort was undertaken in order to identify gaps in the science of HF family caregiving and to outline directions for palliative care researchers. Based on our review of 120 studies that included well over 5700 family caregivers, what has been reported about this population indicates that (1) caregiving situations vary widely with equally wide-ranging tasks for patients to help facilitate their health behaviors, psychological health and relationships and QoL that can consume up to an average of $32 \mathrm{~h} /$ week over 4 years; (2) caregivers have an equally wide range of unmet needs that can fluctuate markedly with patients' unpredictable medical status, are felt to be ignored by the formal healthcare system, and, when unmet, can lead to poor caregiver outcomes including psychological distress, burden, and reduced QoL; and (3) relatively few interventions have been developed and tested that effectively support HF family caregivers. Furthermore, the evidence base shows that unpaid caregivers are an invaluable cornerstone of supportive care to persons with HF, representing a "hidden" lay palliative care workforce who are themselves vulnerable, underserved, and in great need of professional palliative care support. In the following, we interpret the findings in concert with our main topics of review and identify key recommendations for future palliative care research (Boxes 1-4).

Box 1. Recommendations for future study designs, instrumentation, and assessing prevalence and characteristics of the heart failure family caregiver population

1. Conduct more studies using prospective longitudinal designs with larger sample sizes.

2. Increase use of theoretical and/or conceptual models in study question and hypothesis development.
3. Achieve consensus and develop a repository of the most important heart failure caregiver constructs and instruments.

4. Include objective measures, in addition to self-report measures, to broaden the evidence base of the impact of caregiving on caregiver health and functioning (e.g., objective measures of stress, health behaviors, biomarkers).

5. Perform population-based prevalence studies or surveillance practices to ascertain the number, characteristics and responsibilities undertaken by heart failure family caregivers.

6. Develop studies that screen and enroll high-risk heart failure caregivers who are particularly distressed or evidence other unmet needs.

7. Increase focus on understudied family caregiver populations, including carers who are younger, older, male, minorities, working full-time, and nonspouses and multiple caregiver situations.

Box 2. Recommendations for research evaluating the impact of caregivers on patient outcomes

1. Evaluate caregiver's specific direct and indirect impact on more focused areas of patient attitudes, knowledge, and health behaviors (e.g., physical symptom management, advance care planning) using dyadic analysis techniques (e.g., actor-partner interdependence model).

2. Explore the characteristics, quality, and impact of different caregiving strategies have on promoting their care recipients' health behaviors over time.

3. Identify the underlying mechanisms of caregiver characteristics (e.g., strain, mood, relationship history), distinct patient health behaviors (as a process or proximal outcome), and patient quality of life and health (as a primary or distal outcome).

4. Examine benefits and harms of caregivers and the quality of their care on patients' psychological health and relationships, quality of life, and healthcare utilization.

Box 3. Recommendations for research examining the impact of heart failure on the experience and outcomes of caregivers

1. Characterize the impact of caregiving in the last year of life and during after-death grief and bereavement period.

2. Identify successful caregiver coping strategies for distinct situations along the heart failure trajectory (e.g., emergent hospital visits, new symptom or device management).

3. Assess the magnitude of caregiving challenges, coping, and outcomes over the course of heart failure illness.

4. Conduct more in-depth research on specific areas of unmet needs (e.g., managing symptoms, understanding heart failure and prognosis, and handling emergencies).

5. Investigate how and when palliative care can be effectively introduced to patients and families affected by heart failure.

6. Identify factors related to having more difficulty providing care to patients with LVADs and develop interventions to ease the decision-making and transition processes for patients and caregivers.

7. Establish prevalence rates of negative psychological outcomes and risk factors in the caregiver population.

8. Assess the financial and time burden of caregiving across the heart failure trajectory

9. Explore modifiable mechanisms between risk factors and negative outcomes for caregivers 
Box 4. Recommendations for intervention research in heart failure family caregiving

1. Develop and test psychological and psychosocial interventions, with particular attention to the potential role of palliative care specialist clinicians, advance care planning, and dyadic congruence in proxy decisions at end-of-life.

2. Develop comprehensive service models that assess and serve the unique needs of caregivers.

3. Involve heart failure caregivers, patients, clinicians, payers, and other key stakeholders into the intervention development and testing process.

4. Develop pragmatic interventions that can potentially integrate into existing healthcare systems and clinical workflows.

5. Tailor interventions to high-risk subgroups and employ adaptive approaches to intervention content and format.

6. Explore testing of technologies in e- and $\mathrm{m}$-health that might support caregiving tasks.

7. Conduct randomized controlled trials that have sufficient sample sizes and meet quality standards.

8. Implement and refine techniques to boost recruitment and long-term retention of clinical trial participants, especially among minorities and other understudied groups.

9. Gain consensus on primary caregiver outcomes and tools to measure these outcomes.

10. Measure the impact of caregiver intervention on patient outcomes, including utilization and cost-effectiveness.

\section{Research designs, instrumentation, and caregiver characteristics}

The number of studies published on HF family caregiving has steadily increased over the past three decades. The proportion of descriptive, observational research is also slowly lessening relative to other prospective designs and intervention trials (Fig. 1), a trend that indicates a maturing of the evidence base from descriptive knowledge development to intervention development. Despite this, much of the evidence base through 2015 is observational, cross-sectional, lacks a theoretical basis and eligibility criteria, fails to collect or report caregiver demographic data, and is often based on small sample sizes. In combination, this makes it problematic to generalize and confidently recommend clinical practice or policy guidelines for this population. Advancing the science in order to have enough high quality evidence to inform practice and policy will require more adequately powered, theory-based, prospective longitudinal studies.

The majority of measures in HF caregiving research have been self-report measures of general health, QoL, depressive and anxious symptoms, and burden. Moreover, the actual instruments used for each domain vary substantially, making it difficult to draw conclusions regarding the unique impact of caregiving on caregivers. There is also no consensus regarding which domains are important to measure, nor which instruments should be used. Although caregivers' perceptions are useful in understanding the caregiving experience, the overreliance on self-report and the lack of recommendations of measures to use suggest that there is room to advance the science by using other, objective measures of the impact of caregiving and achieving a consensus regarding essential measures and instruments.

Our review of family caregiver characteristics revealed some notable gaps. First, the prevalence and population characteristics of HF family caregivers in the USA (as well as other countries) is unknown. In 2015, the National Alliance for Caregiving published a national profile of US family caregiving for individuals with serious illness and reported that approximately 43.5 million had provided unpaid care to someone in the past 12 months [145]. Of those surveyed, 5\% had reported that "heart disease" was the primary reason for their care recipient needing care (which would equate to 2.2 million caregivers). However, this figure likely represents an underestimate considering the high occurrence of HF as a comorbidity among other serious illnesses tracked in this report. Second, the typical caregiver participant in the reviewed studies was a 55.9-year-old, middle-aged, white, college-educated, female spousal caregiver. Little research to date has focused on caregivers who are young and older adults (65+), minorities, males, full-time employed, less than college educated, and nonspouses and situations where patients have multiple caregivers. Some evidence suggests that these types of caregivers report particularly high burden, thus further raising the importance of conducting studies focused on their experiences. Third, nearly all studies in this review had very general caregiver eligibility criteria (over 20\% reported none) and almost none screened for particularly high-risk caregivers (e.g., high depression, high distress). Future work should seek to characterize and assess high-risk caregivers and their care recipients who may be in particular need of support.

\section{Caregivers' impact on patient}

Just over a quarter of the 120 studies reviewed evaluated caregiver impact on their care recipients' well-being and health. Caregivers' impact on patients' health behaviors was the area most commonly addressed. This relationship was difficult to discern because most studies were cross-sectional and exploratory. Rather, the strength of these studies was in cataloging the vast number of health behaviors for which caregivers provide assistance and further emphasizing the prominent health maintenance role of "lay" home healthcare providers. However, no single health behavior has been studied in depth and hence, future research should consider narrowing the scope to the caregivers' impact on particular patient health behaviors (e.g., physical symptom management, advance care planning). Strikingly, none of the reviewed studies specifically focused on advance care planning as a specific aim. Few 
studies focused on the heart failure family caregiver's impact on their care recipient's psychological health and relationships, QoL, and healthcare utilization especially the important area of resource use at end of life.

Future studies in this area should explore the characteristics, quality, and impact that different strategies used by caregivers to promote their care recipients' health behaviors. Little is also known about the potential mechanisms underlying the relationships among caregiver characteristics (e.g., strain, mood, relationship history), distinct patient health behaviors (as a process or proximal outcome), and patient QoL and health (as a primary or distal outcome). Furthermore, if hospitals, insurers, and other payers of healthcare services are to be persuaded of the benefit of supporting direct formal healthcare services to heart failure family caregivers, it will require evidence of the benefits of the care that caregivers deliver to patients.

\section{Heart failure's impact on caregivers}

Over two thirds of the articles in this review focused on how heart failure affects the experiences, lifestyles, and outcomes of family caregivers. A number of studies, nearly all qualitative, described the family caregiver's experience over the course of the heart failure trajectory, many of which reported consistent themes of adaptation to new and unexpected challenges while at the same time, coping with a sense of uncertainty. A notable absence among these studies was a focus on the time period from when care recipients neared end of life to the after-death grief and bereavement period. Researchers should seek to identify how caregivers navigate the heart failure trajectory by employing longitudinal approaches to assess the changes in challenges, perceived level of control and caregiver outcomes from diagnosis through the bereavement period.

Nearly 30 studies described the number and variety of caregiver needs, highlighting how underserved this population is and the heterogeneity of the role. To capture the individualist nature of the caregiving experience, research targeting the unique unmet needs of caregivers is needed, such as managing symptoms, understanding heart failure and prognosis, and handling emergencies, and interventions to reduce caregiver burden and improve QoL. To address caregivers' concerns over feeling ignored and discounted by the healthcare system, comprehensive models of care that capture the changing needs of caregivers and improve communication between the healthcare team and the caregiver are needed.

A theme throughout several of the studies was how rarely patients and their caregivers sought out and utilized palliative care services [56]. Some studies attributed this to poor prognostic understanding by families and patients, posing a challenge for the introduction and referral to palliative care [42, 55]. Additional challenges included the poor understanding patients and their caregivers had of what palliative care is and the services offered by this specialty [40, 74, 142]. Future work should investigate the best time and process for introducing the option of palliative care to HF patients and their caregivers.

Patients with LVADs present with unique caregiving needs. The studies reviewed here indicate that caregivers need to continually receive informational support during the LVAD decision-making process, which is inherently stressful. Once the LVAD is placed, caregivers are heavily involved in the patient's care, which can be difficult to prepare for and can be associated with caregiver stress. Although evidence suggests that caregivers do accept their roles over time, research should examine factors that may predict which caregivers would have more difficulty adjusting to their roles and interventions to ease the decision-making and transition processes.

Close to 50 studies examined the marked impact that supporting persons with heart failure can have on caregiver's psychological well-being, burden, and QoL. Risk factors for negative caregiver outcomes included a variety of caregiver traits (e.g., being female, having poor health), high caregiving intensity (e.g., more time spent providing care, higher perceived difficulty performing tasks), compromised social functioning (e.g., poor relationship with patients, lack of family support), and care recipients with poorer health. Future work needed in this area is considerable and includes establishing prevalence rates of negative psychological outcomes and risk factors in the caregiver population; evaluating the impact of caregiving in the last year of life as well as into the bereavement period; augmenting the few studies assessing the financial and time burden of caregiving across the heart failure trajectory; and investigating modifiable mechanisms between risk factors and negative outcomes. This research will highlight the magnitude of the issues caregivers face and promote tailored palliative care interventions for those at greatest risk for negative outcomes in the caregiving role.

\section{Heart failure interventions involving caregivers}

Compared to dementia and cancer family caregiving, relatively few interventions have been tested for HF family caregivers and since few high quality trials have been performed, no meta-analyses have been conducted. Interventions reported varied considerably in aims, sample sizes, outcomes assessed and their timing, and methodological rigor. There was also variation in intervention designs and modalities. Of the eight randomized trials, only three reported statistically significant improvements in caregiver outcomes, suggesting the need for more experimentation in intervention design, although many of these trials had considerably low power. Therefore, greater priority should be given to intervention development and clinical trials testing in this area. 
We offer several recommendations for $\mathrm{HF}$ family caregiver intervention research. We recommend involving HF caregivers, patients, clinicians, payers, and other key stakeholders into co-designing interventions. Pragmatic intervention testing is also warranted that can potentially integrate into existing healthcare systems and clinical workflows. There is a need for tailoring interventions to high-risk caregiver subgroups (e.g., rural-dwellers, caring for patients with limited functional mobility or with depression) and employ adaptive approaches to intervention content and format. Given the wide variability of caregiving situations and needs (e.g., spouses may need different support from children, in-home caregivers may differ from commuting caregivers), an adaptive intervention strategy may help target intervention content or types of support to different caregiver needs. There are also unexplored possibilities in leveraging e- and m-health technologies that might support caregiving tasks. We also strongly advocate for the conduct of high quality, sufficiently powered randomized controlled trials that meet CONSORT reporting [146] and other quality standards $[147,148]$. To this end, techniques of recruitment and retention of HF family caregiver participants will need to be refined, especially those that target minorities and other understudied groups. To help facilitate future metaanalyses of clinical trials, we believe that consensus is needed on primary outcomes of interest and tools to measures these outcomes. Future interventions should also test the value added of including specialist palliative care clinicians into models of family caregiver support as well as develop strategies of advance care planning, potentially targeting communication skills and dyadic congruence. Finally, measuring the impact of caregiver interventions on patient outcomes including utilization and cost-effectiveness will be necessary to persuade payers of the value in directly supporting HF family caregiving.

\section{Implications for palliative care researchers}

Family caregivers are recognized in the National Consensus Statement as well as by other national and international palliative care professional organizations, as part of the unit of care that is the primary focus of palliative care clinical practice and research $[10,149,150]$. The aforementioned research recommendations are thus of principal relevance to the palliative care research community and should serve as priority areas of focus over the next decade as the prevalence of symptomatic advanced HF continues to rise [151]. Furthermore, researchers specializing in developing and testing interventions should explore models of care delivery that entail palliative care specialists performing comprehensive assessment and direct support to HF family caregivers as similar models of such care in oncology have shown promising results $[152,153]$. It will also be important for palliative care researchers to prospectively test whether palliative care support of HF family caregivers leads to improvements in patient well-being and healthcare utilization, particularly as end-of-life nears. Such work can help guide payment reform that might one day reimburse for direct services to family caregivers as insurers and healthcare systems shift to value-based and bundled payment reimbursement [154]. As palliative care looks forward to continued service growth, we encourage palliative care researchers to take the lead on research and clinical practice expansion in the family caregiving space [155].

\section{Limitations}

This review has several limitations. First, our search was limited to articles indexed in PubMed and CINAHL; hence, there may be studies that were inadvertently missed. While this is certainly possible, our aim was to ascertain scientific gaps in the overall evidence base and we believe that the articles included here were sufficient to represent the "population" of existing articles that compose what we know about HF family caregivers. Second, we did not include studies published in languages other than English, unpublished studies, dissertations, or abstracts from conference proceedings. Third, we did not systematically evaluate the quality of the research designs for each study. Such a task was beyond the resources available given the scope of this review; future reviews that are more focused in their aims would undoubtedly yield stronger and more nuanced conclusions from engaging in such an effort.

\section{Conclusions}

Most of the 23 million individuals with HF worldwide receive daily, unpaid support from close family members and friends. By 2030, the number of individuals with HF is expected to increase to over eight million, or 1 out of every 33 persons [156]. Family caregivers will be relied upon to provide the majority of home care to these individuals [157] as US family caregiving has been valued at $\$ 522$ billion per year [158], making it unlikely that these services can be replaced with paid professionals. We believe a fundamental paradigm shift is needed that recognizes HF caregivers as co-providers and co-recipients of care alongside patients. We call on palliative care researchers, policy makers, payers, and HF and palliative care funding entities, such as the American Heart Association, the National Heart, Lung, and Blood Institute, and the National Institute of Nursing Research, to address the research gaps identified in this review and support forward progress in the science of HF family caregiving.

Acknowledgments The authors would like to gratefully acknowledge Allison Monaco, Jakira Myers, and Amanda Segars for assisting with study abstraction. Dr. Dionne-Odom is supported by the National Institute for Nursing Research (1K99NR015903), the National 
Palliative Care Research Center, and the American Cancer Society (RSG PCSM - 124668). Dr. Bekelman is funded by VA HSR\&D IIR 14-346. Dr. Ejem is supported by a Research Supplement to Promote Diversity in Health Research (R01 NR013665-01A1). Ms. Wells is supported by a Robert Wood Johnson Foundation Future of Nursing Scholarship. Dr. Strömberg is supported by the Swedish Research Council for Health, Working Life and Welfare (2014-4100), and the Swedish Research Council for Medicine and Health (K2015-99X-22124-04-4). Dr. Evangelista is supported by the National Heart, Lung, and Blood Institute (1R01HL093466-01). Dr. Bakitas is supported by the National Institute for Nursing Research (R01 NR013665-01A1) and the American Cancer Society (\#RSG PCSM - 124668).

\section{Compliance with ethical standards}

Conflict of interest The authors declare that they have no conflict of interest.

\section{References}

1. Bui AL, Horwich TB, Fonarow GC (2011) Epidemiology and risk profile of heart failure. Nat Rev Cardiol 8(1):30-41

2. Pulignano $\mathrm{G}$ et al (2016) Atrial fibrillation, cognitive impairment, frailty and disability in older heart failure patients. J Cardiovasc Med (Hagerstown) 17(8):616-623

3. Lum HD et al (2016) Burdensome physical and depressive symptoms predict heart failure-specific health status over one year. J Pain Symptom Manag 51(6):963-970

4. Lainscak M et al (2011) Self-care management of heart failure: practical recommendations from the patient Care Committee of the Heart Failure Association of the European Society of Cardiology. Eur J Heart Fail 13(2):115-126

5. Riegel B et al (2009) State of the science: promoting self-care in persons with heart failure: a scientific statement from the American Heart Association. Circulation 120(12):1141-1163

6. Institute of Medicine (2014) Dying in America: improving quality and honoring individual preferences near the end of life. Washington, DC

7. Institute of Medicine (2012) Living well with chronic illness: a call for public health action. Washington, DC

8. Institute of Medicine (2008) Retooling for an aging America: building the health care workforce. Washington, DC.

9. National Academies of Sciences, E., and Medicine (2016) In: Schulz R, Eden J (eds) Families caring for an aging America. The National Academies Press, Washington, DC

10. National Consensus Project (2013) Clinical practice guidelines for quality palliative care. Pittsburgh, PA

11. Braun LT, et al. (2016) Palliative care and cardiovascular disease and stroke: a policy statement from the American Heart Association/American Stroke Association. Circulation

12. Kang X, Li Z, Nolan MT (2011) Informal caregivers' experiences of caring for patients with chronic heart failure: systematic review and metasynthesis of qualitative studies. J Cardiovasc Nurs 26(5): 386-394

13. Hooker SA et al (2015) The impact of relationship quality on health-related outcomes in heart failure patients and informal family caregivers: an integrative review. J Cardiovasc Nurs 30(4 Suppl 1):S52-S63

14. Doherty LC, Fitzsimons D, McIlfatrick SJ (2016) Carers' needs in advanced heart failure: a systematic narrative review. Eur J Cardiovasc Nurs 15(4):203-212
15. Strömberg A, Luttik ML (2015) Burden of caring: risks and consequences imposed on caregivers of those living and dying with advanced heart failure. Curr Opin Support Palliat Care 9(1):26-30

16. Whittingham K, Barnes S, Gardiner C (2013) Tools to measure quality of life and carer burden in informal carers of heart failure patients: a narrative review. Palliat Med 27(7):596-607

17. Clark AM et al (2014) Determinants of effective heart failure selfcare: a systematic review of patients' and caregivers' perceptions. Heart 100(9):716-721

18. Buck HG et al (2015) Caregivers' contributions to heart failure self-care: a systematic review. Eur J Cardiovasc Nurs 14(1):79-89

19. Dunbar SB et al (2008) Family influences on heart failure self-care and outcomes. J Cardiovasc Nurs 23(3):258-265

20. Magid M et al (2016) The perceptions of important elements of caregiving for a left ventricular assist device patient: a qualitative meta-synthesis. J Cardiovasc Nurs 31(3):215-225

21. Dixon-Woods $\mathrm{M}$ et al (2005) Synthesising qualitative and quantitative evidence: a review of possible methods. J Health Serv Res Policy 10(1):45-53

22. Aggarwal B et al (2015) Factors associated with medication adherence among heart failure patients and their caregivers. J Nurs Educ Pract 5(3):22-27

23. Ågren S et al (2011) The influence of chronic heart failure in patient-partner dyads - a comparative study addressing issues of health-related quality of life. J Cardiovasc Nurs 26(1):65-73

24. Agren S, Evangelista L, Stromberg A (2010) Do partners of patients with chronic heart failure experience caregiver burden? Eur J Cardiovasc Nurs 9(4):254-262

25. Ågren S et al (2012) Dyads affected by chronic heart failure: a randomized study evaluating effects of education and psychosocial support to patients with heart failure and their partners. J Card Fail 18(5):359-366

26. Ågren S et al (2015) Caregiving tasks and caregiver burden; effects of an psycho-educational intervention in partners of patients with post-operative heart failure. Heart Lung 44(4):270-275

27. Akbarin M, Aarts C (2013) Being a close relative of a patient with a left ventricular assist device. Eur J Cardiovasc Nurs 12(1):64-68

28. Aldred H, Gott M, Gariballa S (2005) Advanced heart failure: impact on older patients and informal carers. J Adv Nurs 49(2): 116-124

29. Annema C, Luttik ML, Jaarsma T (2009) Reasons for readmission in heart failure: perspectives of patients, caregivers, cardiologists, and heart failure nurses. Heart Lung 38(5):427-434

30. Bahrami $\mathrm{M}$ et al (2014) Informational needs and related problems of family caregivers of heart failure patients: a qualitative study. J Educ Health Promot 3:113

31. Bahrami $\mathrm{M}$ et al (2014) Caregiver burden among Iranian heart failure family caregivers: a descriptive, exploratory, qualitative study. Iran J Nurs Midwifery Res 19(1):56-63

32. Bakas T et al (2006) Family caregiving in heart failure. Nurs Res 55(3):180-188

33. Baker $\mathrm{K}$ et al (2010) Caregiving for patients requiring left ventricular assistance device support. Heart Lung 39(3):196-200

34. Barber J, Leslie G (2015) A simple education tool for ventricular assist device patients and their caregivers. J Cardiovasc Nurs 30(3):E1-E10

35. Barnes $\mathrm{S}$ et al (2006) Characteristics and views of family carers of older people with heart failure. Int J Palliat Nurs 12(8):380-389

36. Bekelman DB et al (2011) Giving voice to patients' and family caregivers' needs in chronic heart failure: implications for palliative care programs. J Palliat Med 14(12):1317-1324

37. Bidwell JT et al (2015) Determinants of heart failure self-care maintenance and Management in Patients and Caregivers: a dyadic analysis. Res Nurs Health 38(5):392-402

38. Blumenthal-Barby JS et al (2015) Assessment of patients' and caregivers' informational and decisional needs for left ventricular 
assist device placement: implications for informed consent and shared decision-making. J Heart Lung Transplant 34(9):11821189

39. Boyd KJ et al (2004) Living with advanced heart failure: a prospective, community based study of patients and their carers. Eur J Heart Fail 6(5):585-591

40. Boyd KJ et al (2009) Making sure services deliver for people with advanced heart failure: a longitudinal qualitative study of patients, family carers, and health professionals. Palliat Med 23(8):767776

41. Brännström $\mathrm{M}$ et al (2007) Being a close relative of a person with severe, chronic heart failure in palliative advanced home care-a comfort but also a strain. Scand J Caring Sci 21(3):338-344

42. Browne $\mathrm{S}$ et al (2014) Patient, carer and professional perspectives on barriers and facilitators to quality care in advanced heart failure. PLoS One 9(3):e93288

43. Buck HG, Kitko L, Hupcey JE (2013) Dyadic heart failure care types: qualitative evidence for a novel typology. J Cardiovasc Nurs 28(6):E37-E46

44. Buck HG et al (2015) Exploring the relationship of patient and informal caregiver characteristics with heart failure self-care using the actor-partner interdependence model: implications for outpatient palliative care. J Palliat Med 18(12):1026-1032

45. Buck HG, et al. (2013) Everything they were discussing, we were already doing: hospice heart failure caregivers reflect on a palliative caregiving intervention. J Hosp Palliat Nurs 15(4)

46. Buck HG, Zambroski CH, Hupcey JE (2013) Assessing the response of patients and spousal/partner caregivers to a new instrument measuring dyadic heart failure care types. Eur J Cardiovasc Nurs 12(2):209-213

47. Bull MJ, Hansen HE, Gross CR (2000) Predictors of elder and family caregiver satisfaction with discharge planning. J Cardiovasc Nurs 14(3):76-87

48. Burke RE et al (2014) Caregivers' perceived roles in caring for patients with heart failure: what do clinicians need to know? J Card Fail 20(10):731-738

49. Burton AM et al (2012) Burden and well-being among a diverse sample of cancer, congestive heart failure, and chronic obstructive pulmonary disease caregivers. J Pain Symptom Manag 44(3):410 420

50. Chiang LC et al (2012) The effectiveness of telehealth care on caregiver burden, mastery of stress, and family function among family caregivers of heart failure patients: a quasi-experimental study. Int J Nurs Stud 49(10):1230-1242

51. Chung ML et al (2009) The effects of depressive symptoms and anxiety on quality of life in patients with heart failure and their spouses: testing dyadic dynamics using actor-partner interdependence model. J Psychosom Res 67(1):29-35

52. Chung ML et al (2010) Predictors of depressive symptoms in caregivers of patients with heart failure. J Cardiovasc Nurs 25(5):411-419

53. Clark AM et al (2009) Patient and informal caregivers' knowledge of heart failure: necessary but insufficient for effective self-care. Eur J Heart Fail 11(6):617-621

54. Clark AM et al (2008) The complex nature of informal care in home-based heart failure management. J Adv Nurs 61(4):373-383

55. Clark PC, Dunbar SB (2003) Family partnership intervention: a guide for a family approach to care of patients with heart failure. AACN Clin Issues 14(4):467-476

56. Davidson PM et al (2013) The caregiving perspective in heart failure: a population based study. BMC Health Serv Res 13:342

57. Delgado JF et al (2015) Health-related quality of life, social support, and caregiver burden between six and 120 months after heart transplantation: a Spanish multicenter cross-sectional study. Clin Transpl 29(9):771-780
58. Dionne-Odom JN, et al. (2014) Translating and testing the ENABLE: CHF-PC concurrent palliative care model for older adults with heart failure and their family caregivers. J Palliat Med

59. Dracup K et al (2004) Emotional well-being in spouses of patients with advanced heart failure. Heart Lung 33(6):354-361

60. Egerod I, Overgaard D (2012) Taking a back seat: support and self-preservation in close relatives of patients with left ventricular assist device. Eur J Cardiovasc Nurs 11(4):380-387

61. Etemadifar $\mathrm{S}$ et al (2015) Family caregivers' experiences of caring for patients with heart failure: a descriptive, exploratory qualitative study. J Nurs Res 23(2):153-161

62. Etemadifar $\mathrm{S}$ et al (2014) The effectiveness of a supportive educative group intervention on family caregiver burden of patients with heart failure. Iran J Nurs Midwifery Res 19(3):217-223

63. Evangelista LS et al (2002) Emotional well-being of heart failure patients and their caregivers. J Card Fail 8(5):300-305

64. Foebel AD, Hirdes JP, Heckman GA (2012) Caregiver status affects medication adherence among older home care clients with heart failure. Aging Clin Exp Res 24(6):718-721

65. Freydberg $\mathrm{N}$ et al (2010) "If he gives in, he will be gone...": the influence of work and place on experiences, reactions and selfcare of heart failure in rural Canada. Soc Sci Med 70(7):10771083

66. Gure TR et al (2008) Degree of disability and patterns of caregiving among older Americans with congestive heart failure. J Gen Intern Med 23(1):70-76

67. Harding R et al (2008) Meeting the communication and information needs of chronic heart failure patients. J Pain Symptom Manag 36(2):149-156

68. Holden RJ, Schubert CC, Mickelson RS (2015) The patient work system: an analysis of self-care performance barriers among elderly heart failure patients and their informal caregivers. Appl Ergon 47:133-150

69. Hooley PJ, Butler G, Howlett JG (2005) The relationship of quality of life, depression, and caregiver burden in outpatients with congestive heart failure. Congest Heart Fail 11(6):303-310

70. Hopp FP et al (2014) Go to the hospital or stay at home? A qualitative study of expected hospital decision making among older African Americans with advanced heart failure. J Gerontol Soc Work 57(1):4-23

71. Hunting $\mathrm{G}$ et al (2015) A multi-level qualitative analysis of Telehomecare in Ontario: challenges and opportunities. BMC Health Serv Res 15:544

72. Hupcey JE et al (2011) Palliative needs of spousal caregivers of patients with heart failure followed at specialized heart failure centers. J Hosp Palliat Nurs 13(3):142-150

73. Hupcey JE et al (2010) Achieving medical stability: wives' experiences with heart failure. Clin Nurs Res 19(3):211-229

74. Hupcey JE, Penrod J, Fogg J (2009) Heart failure and palliative care: implications in practice. J Palliat Med 12(6):531-536

75. Hwang B, Fleischmann KE, Howie-Esquivel J, Stotts NA, Dracup $\mathrm{K}$ (2011) Caregiving for patients with heart failure: impact on patients' families. Am J Crit Care 20(6):431-441

76. Hwang B et al (2010) Family caregiving for patients with heart failure: types of care provided and gender differences. J Card Fail 16(5):398-403

77. Imes CC et al (2011) Descriptive study of partners' experiences of living with severe heart failure. Heart Lung 40(3):208-216

78. Jones J et al (2015) The future as a series of transitions: qualitative study of heart failure patients and their informal caregivers. J Gen Intern Med 30(2):176-182

79. Joo $\mathrm{H}$ et al (2015) Cost of informal caregiving for patients with heart failure. Am Heart J 169(1):142-48.e2

80. Kaholokula JK et al (2008) Pacific Islanders' perspectives on heart failure management. Patient Educ Couns 70(2):281-291 
81. Karmilovich SE (1994) Burden and stress associated with spousal caregiving for individuals with heart failure. Prog Cardiovasc Nurs 9(1):33-38

82. Kirkpatrick JN et al (2015) Caregivers and left ventricular assist devices as a destination, not a journey. J Card Fail 21(10):806-815

83. Kitko LA, Hupcey JE (2013) The work of spousal caregiving of older adults with end-stage heart failure. J Gerontol Nurs 39(7): 40-47

84. Kitko LA et al (2013) Caring for a spouse with end-stage heart failure through implantation of a left ventricular assist device as destination therapy. Heart Lung 42(3):195-201

85. Kitko LA et al (2015) Patient and caregiver incongruence in advanced heart failure. Clin Nurs Res 24(4):388-400

86. Lee CS et al (2015) Patterns and predictors of patient and caregiver engagement in heart failure care: a multi-level dyadic study. Int J Nurs Stud 52(2):588-597

87. Levin JB et al (2014) Health literacy and heart failure management in patient-caregiver dyads. J Card Fail 20(10):755-761

88. Liljeroos $\mathrm{M}$ et al (2014) Perceived caring needs in patient-partner dyads affected by heart failure: a qualitative study. J Clin Nurs 23(19-20):2928-2938

89. Löfvenmark C et al (2013) Evaluation of an educational programme for family members of patients living with heart failure: a randomised controlled trial. J Clin Nurs 22(1-2):115-126

90. Lum HD et al (2014) Caregiving in heart failure: relationship quality is associated with caregiver benefit finding and caregiver burden. Heart Lung 43(4):306-310

91. Luttik ML et al (2007) Living with heart failure: partner perspectives. J Cardiovasc Nurs 22(2):131-137

92. Luttik ML et al (2009) Quality of life in partners of people with congestive heart failure: gender and involvement in care. J Adv Nurs 65(7):1442-1451

93. Luttik ML et al (2007) Caregiver burden in partners of heart failure patients; limited influence of disease severity. Eur J Heart Fail 9(6-7):695-701

94. Luttik ML et al (2005) For better and for worse: quality of life impaired in HF patients as well as in their partners. Eur $\mathrm{J}$ Cardiovasc Nurs 4(1):11-14

95. Lyons KS et al (2015) A dyadic approach to managing heart failure with confidence. J Cardiovasc Nurs 30(4 Suppl 1):S64-S71

96. Malik FA, Gysels M, Higginson IJ (2013) Living with breathlessness: a survey of caregivers of breathless patients with lung cancer or heart failure. Palliat Med 27(7):647-656

97. Marcuccilli L et al (2014) Family caregivers' inside perspectives: caring for an adult with a left ventricular assist device as a destination therapy. Prog Transplant 24(4):332-340

98. Mårtensson J et al (2003) Living with heart failure: depression and quality of life in patients and spouses. J Heart Lung Transplant 22(4):460-467

99. McIlvennan CK et al (2015) Decision-making for destination therapy left ventricular assist devices: implications for caregivers. Circ Cardiovasc Qual Outcomes 8(2):172-178

100. McMillan SC, et al. (2013) The COPE intervention for caregivers of patients with heart failure: an adapted intervention. J Hosp Palliat Nurs 15(4)

101. Molloy GJ et al (2006) Effects of an exercise intervention for older heart failure patients on caregiver burden and emotional distress. Eur J Cardiovasc Prev Rehabil 13(3):381-387

102. Molloy GJ et al (2008) Using the demand-control model of job strain to predict caregiver burden and caregiver satisfaction in the informal caregivers of heart failure patients. Br J Health Psychol 13(Pt 3):401-417

103. Moore C (2010) The impact of family functioning on caregiver burden among caregivers of veterans with congestive heart failure. J Fam Soc Work 13(5):451-462
104. Murray SA et al (2004) Exploring the spiritual needs of people dying of lung cancer or heart failure: a prospective qualitative interview study of patients and their carers. Palliat Med 18(1): $39-45$

105. Negarandeh $R$ et al (2015) The relationship between perceived life changes and mental health in family caregivers of patients with heart failure who referred to Rajaei Cardiovascular Medical and Research Center, Tehran. Int J Community Based Nurs Midwifery 3(4):283-291

106. Pattenden JF, Roberts H, Lewin RJ (2007) Living with heart failure; patient and carer perspectives. Eur J Cardiovasc Nurs 6(4): 273-279

107. Piamjariyakul U et al (2013) The feasibility of a telephone coaching program on heart failure home management for family caregivers. Heart Lung 42(1):32-39

108. Piamjariyakul U et al (2012) Part 2: enhancing heart failure home management: integrated evidence for a new family caregiver educational plan. Appl Nurs Res 25(4):246-250

109. Piamjariyakul U et al (2012) Part I: heart failure home management: patients, multidisciplinary health care professionals and family caregivers' perspectives. Appl Nurs Res 25(4):239-245

110. Piamjariyakul U et al (2015) Caregiver coaching program effect: reducing heart failure patient rehospitalizations and improving caregiver outcomes among African Americans. Heart Lung 44(6):466-473

111. Piette JD et al (2008) Improving heart failure self-management support by actively engaging out-of-home caregivers: results of a feasibility study. Congest Heart Fail 14(1):12-18

112. Piette JD et al (2015) A randomized trial of mobile health support for heart failure patients and their informal caregivers: impacts on caregiver-reported outcomes. Med Care 53(8):692-699

113. Pressler SJ et al (2013) Family caregivers of patients with heart failure: a longitudinal study. J Cardiovasc Nurs 28(5):417-428

114. Pressler SJ et al (2009) Family caregiver outcomes in heart failure. Am J Crit Care 18(2):149-159

115. Quinn C, Dunbar SB, Higgins M (2010) Heart failure symptom assessment and management: can caregivers serve as proxy? J Cardiovasc Nurs 25(2):142-148

116. Retrum JH, Nowels CT, Bekelman DB (2013) Patient and caregiver congruence: the importance of dyads in heart failure care. $\mathrm{J}$ Cardiovasc Nurs 28(2):129-136

117. Ross L, Austin J (2015) Spiritual needs and spiritual support preferences of people with end-stage heart failure and their carers: implications for nurse managers. J Nurs Manag 23(1):87-95

118. Sanford J et al (2011) A process of decision making by caregivers of family members with heart failure. Res Theory Nurs Pract 25(1):55-70

119. Saunders MM (2012) Perspectives from family caregivers receiving home nursing support: findings from a qualitative study of home care patients with heart failure. Home Healthc Nurse 30(2):82-90

120. Saunders MM (2010) Working and caregiving: a comparison of employed and unemployed caregivers of older heart failure patients. Holist Nurs Pract 24(1):16-22

121. Saunders MM (2009) Indicators of health-related quality of life in heart failure family caregivers. J Community Health Nurs 26(4): 173-182

122. Saunders MM (2008) Family caregiver support and hospitalizations of patients with heart failure. Home Healthc Nurse 26(10): 624-632

123. Saunders MM (2008) Factors associated with caregiver burden in heart failure family caregivers. West J Nurs Res 30(8):943-959

124. Schwarz KA, Dunphy G (2003) An examination of perceived stress in family caregivers of older adults with heart failure. Exp Aging Res 29(2):221-235 
125. Schwarz KA, Elman CS (2003) Identification of factors predictive of hospital readmissions for patients with heart failure. Heart Lung 32(2):88-99

126. Schwarz KA et al (2008) Telemonitoring of heart failure patients and their caregivers: a pilot randomized controlled trial. Prog Cardiovasc Nurs 23(1):18-26

127. Scott LD (2000) Caregiving and care receiving among a technologically dependent heart failure population. ANS Adv Nurs Sci 23(2):82-97

128. Sebern M, Riegel B (2009) Contributions of supportive relationships to heart failure self-care. Eur J Cardiovasc Nurs 8(2):97-104

129. Sebern MD, Woda A (2012) Shared care dyadic intervention: outcome patterns for heart failure care partners. West J Nurs Res 34(3):289-316

130. Shahriari $\mathrm{M}$ et al (2013) Effects of a family support program on self-care behaviors in patients with congestive heart failure. Iran J Nurs Midwifery Res 18(2):152-157

131. Small N et al (2009) Dying, death and bereavement: a qualitative study of the views of carers of people with heart failure in the UK. BMC Palliat Care 8:6

132. Sneed NV, Finch NJ, Leman RB (1994) The impact of device recall on patients and family members of patients with automatic implantable cardioverter defibrillators. Heart Lung 23(4):317-322

133. Trivedi RB et al (2012) Examining the interrelatedness of patient and spousal stress in heart failure: conceptual model and pilot data. J Cardiovasc Nurs 27(1):24-32

134. Vellone E et al (2014) Effects of self-care on quality of life in adults with heart failure and their spousal caregivers: testing dyadic dynamics using the actor-partner interdependence model. J Fam Nurs 20(1):120-141

135. Vellone E et al (2015) The key role of caregiver confidence in the caregiver's contribution to self-care in adults with heart failure. Eur J Cardiovasc Nurs 14(5):372-381

136. Walden JA et al (2001) Educational needs of patients with advanced heart failure and their caregivers. J Heart Lung Transplant 20(7):766-769

137. Whittingham K, Pearce DE (2011) Carer support from a community-based heart specialist nurse service. Br J Nurs 20(21):1388-1393

138. Wingham J et al (2015) Needs of caregivers in heart failure management: a qualitative study. Chronic Illn 11(4):304-319

139. Yeh PM, Bull M (2012) Use of the resiliency model of family stress, adjustment and adaptation in the analysis of family caregiver reaction among families of older people with congestive heart failure. Int J Older People Nursing 7(2):117-126

140. Young LE et al (2010) Families and heart transplantation: reversing the trajectory of end stage heart disease. Can J Cardiovasc Nurs 20(2):6-17

141. Bozkurt Zincir S et al (2014) Burden and depressive symptoms associated with adult-child caregiving for individuals with heart failure. ScientificWorldJournal 2014:641817
142. Harding R et al (2008) Meeting the communication and information needs of chronic heart failure patients. J Pain Symptom Manag 36(2):149-156

143. Bakas $\mathrm{T}$ et al (2006) Psychometric testing of the revised 15-item Bakas Caregiving Outcomes Scale. Nurs Res 55(5):346-355

144. Burton AM et al (2008) Predictors of well-being in bereaved former hospice caregivers: the role of caregiving stressors, appraisals, and social resources. Palliat Support Care 6(2):149-158

145. Institute N.A.f.C.a.A.P.P (2015) Caregiving in the U.S. Washington DC

146. Schulz KF et al (2010) CONSORT 2010 statement: updated guidelines for reporting parallel group randomised trials. BMJ 340:c332

147. Chan AW et al (2013) SPIRIT 2013 statement: defining standard protocol items for clinical trials. Ann Intern Med 158(3):200-207

148. Hoffmann TC et al (2014) Better reporting of interventions: template for intervention description and replication (TIDieR) checklist and guide. BMJ 348:g1687

149. National Quality Forum (2006) A national framework and preferred practices for palliative and hospice care quality: a consensus report. National Quality Forum: Washington, DC

150. Payne S, EAPC Task Force on Family Carers (2010) White paper on improving support for family carers in palliative care: part 1. Eur J Palliat Care 17(5):238-245

151. Mozaffarian D et al (2016) Heart disease and stroke statistics-2016 update: a report from the American Heart Association. Circulation 133(4):e38-e60

152. Dionne-Odom JN, et al. (2015) Benefits of early versus delayed palliative care to informal family caregivers of patients with advanced cancer: outcomes from the ENABLE III randomized controlled trial. J Clin Oncol

153. Sun V et al (2015) Effectiveness of an interdisciplinary palliative care intervention for family caregivers in lung cancer. Cancer 121(20):3737-3745

154. Parikh RB, et al. (2016) Scalable principles of community-based high-value care for seriously ill individuals: diamonds in the rough. Healthc (Amst)

155. Kamal AH, Dionne-Odom JN (2016) A Blue Ocean strategy for palliative care: focus on family caregivers. J Pain Symptom Manag 51(3):e1-e3

156. Heidenreich PA et al (2013) Forecasting the impact of heart failure in the United States: a policy statement from the American Heart Association. Circ Heart Fail 6(3):606-619

157. Roth DL, Fredman L, Haley WE (2015) Informal caregiving and its impact on health: a reappraisal from population-based studies. Gerontologist 55(2):309-319

158. Chari AV et al (2015) The opportunity costs of informal elder-care in the United States: new estimates from the American time use survey. Health Serv Res 50(3):871-882 